\title{
Kernos
}

Revue internationale et pluridisciplinaire de religion grecque antique

9| 1996

Varia

\section{Ugo Bianchi (1922-1995)}

L'histoire de la religion grecque comme histoire des religions

\section{Giovanni Casadio}

\section{(2) OpenEdition}

1 Journals

\section{Édition électronique}

URL : http://journals.openedition.org/kernos/1151

DOI : 10.4000/kernos. 1151

ISSN : 2034-7871

Éditeur

Centre international d'étude de la religion grecque antique

Édition imprimée

Date de publication : 1 janvier 1996

ISSN : 0776-3824

Référence électronique

Giovanni Casadio, « Ugo Bianchi (1922-1995) », Kernos [En ligne], 9 | 1996, mis en ligne le 20 avril 2011, consulté le 30 avril 2019. URL : http://journals.openedition.org/kernos/1151 


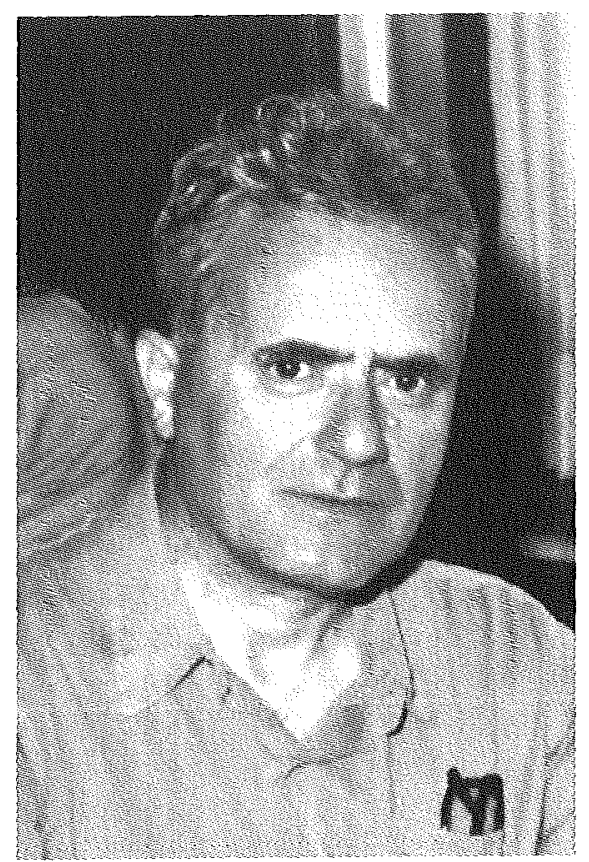




\title{
Ugo Bianchi (1922-1995)
}

\section{L'histoire de la religion grecque comme histoire des religions}

\author{
par Giovanni CASADIO
}

Le premier livre et le premier article d'Ugo Bianchi traitaient des problèmes importants de la religion grecque, et sa thèse de licence en 1944 était consacrée à une déesse grecque, Artémis. Toutefois, dans la variété multiforme de sa production, les recherches se rapportant à la religion grecque ne semblent pas l'emporter sur celles dédiées aux autres domaines culturels qui lui étaient familiers : l'Iran, l'Italie, le domaine judéo-chrétien, l'Orient ancien, la période hellénistique, l'ethnologie. Et aucune des entreprises collectives projetées et animées par lui (avec la publication réussie d'une bonne dizaine de volumes d'Actes) ne fut exclusivement dédiée à la religion grecque. En fait, pour Bianchi, comme pour son maître Raffaele Pettazzoni, la religion grecque en tant que composante de la culture grecque n'existait pas. Pour lui existait seulement l'histoire des religions, ou plutôt le problème capital de l'histoire des religions : d'où venons-nous? qui sommes-nous? où allons-nous ? En d'autres termes, il a toujours agité le problème des rapports entre « l'homme, le dieu et la destinée » : ainsi, dans le sous-titre de sa première monographie (formule reprise dans l'incipit d'une mise au point de 1989: « Man, God and Destiny: this is a central issue of religious, and generally human concern » [p. 11]). La même triade conceptuelle - mais exprimée au niveau d'une praxis éthique d'un point de vue anthropocentrique - dans le sous-titre d'un résumé didactique publié en 1976, dont le triple intérêt apparaît dans le titre (Prometeo, Orfeo, Adamo) : « la destinée, le mal, le salut ». Par contre, l'histoire des religions anciennes dans son ensemble est revécue et réinterprétée à la lumière d'exemples fournis par la religion grecque, ou plutôt la philosophie religieuse des Grecs (la Religiosität de W. Nestle). Cela ne signifie pas que le savant aimait la philosophie en tant que discipline avec son esprit de système et ses bavardages auto-référentiels. Comme ses élèves et les lecteurs de ses ouvrages de méthodologie le savent, Bianchi, même s'il l'honorait, reléguait la philosophie de la religion hors du domaine des sciences religieuses. Pourtant, au centre de toute sa vision historico-religieuse, il y avait un philosophe - et quel philosophe ! - qui lui donnait la clef interprétative du passage des formes de religiosité ethnique aux théologies évoluées de l'Axenzeit jusqu'aux systèmes gnostiques de la Zeitwende. En fait, plus que la philosophie, le professeur Bianchi appréciait l'érudition et, contrairement aux apparences, l'érudition 
archéologique encore davantage que l'érudition philologique. Lui était étrangère toute espèce d'herméneutique historico-littéraire ou historico-artistique dont la seule importance était esthétique : une conséquence de son éducation profondément positiviste - en antithèse avec le climat idéaliste instauré dans l'Italie de l'entre-deux-guerres par Croce et Gentile. Ce n'est pas un hasard s'ils furent deux adversaires irréductibles de l'histoire des religions en tant que discipline autonome. Bianchi était en accord, au contraire, avec les maîtres de son Alma Mater : Pettazzoni, Lugli, Cardinali, Furlani, Guarducci, Blanc, Funaioli (un peu moins avec son professeur de littérature grecque, G. Perrotta, pour lequel cependant il nourrissait sympathie et admiration). Mises à part les sections érudites de la monographie de 1953 (le chapitre sur «Moira nella religione dei Greci », p. 193-220, à propos duquel D. Sabbatucci, in SMSR, 24-25 [19531954], p. 266-268, parlait de «unilateralità » et relevait les carences de la documentation), dans le manuel de 1975 (le chapitre concernant « La religione minoico-micenea », p. 3-36, qui est un abrégé critique de Nilsson avec quelques approfondissements remarquables) et dans le répertoire iconographique de 1976 (dans les légendes des illustrations que cependant C. Bérard, in Gnomon, 1978, p. 374-375, juge bien décevantes), la disposition - et non une véritable vocation - pour l'érudition minutieuse et purement descriptive ne se révèle que dans une des rares enquêtes concernant un thème cultuel en dehors du pôle de réflexion déjà mentionné : l'étude sur Athana Lindia (1957), qui semble en effet un travail de commande (finalisé pour l'article dans le $D E A R$ ), dans lequel on perçoit l'inspiration de G. Cardinali bien plus que celle de R. Pettazzoni.

Dans Dios aisa (1953), qui lui servit pour obtenir la « libera docenza » en histoire des religions (1954), on trouve in nuce maintes idées maîtresses qui féconderont la suite de sa recherche, focalisée précisément sur le thème de la destinée de l'homme dans cette vie et dans l'autre, en relation avec l'altétité divine. La richesse des questions posées et des réponses données (pas toujours convaincantes, mais toujours intelligentes) est presque inépuisable, mais un style foisonnant, typique des travaux de jeunesse, ne les rend pas nécessairement accessibles. Il faut remarquer que ce livre suscita immédiatement l'attention de Nilsson (qui, dans Gnomon, 1954, p. 480-482, en souligna les mérites) et qu'il est devenu ensuite un point de repère obligé dans les discussions sur le sujet (de B.C. Dietrich à H. Erbse). La Religione greca (1975), au contraire, représente une somme des recherches menées sur les thèmes qui intéressaient l'auteur : en conséquence, elle est brillante sur ces thèmes et assez faible pour le reste, surtout les institutions cultuelles relatives à la religion olympienne, non mystique ( $c f$. A. Brelich dans le c.r. de la première édition [1962], in SMSR, 34 [1963], p. 271, qui en reconnaissait pourtant les éminentes qualités). Remarquablement développée est la partie sur la religion hellénistique où le mysticisme, le syncrétisme, la sotétiologie individuelle (soit les aspects de la religion qui l'intéressaient) sont prédominants. 
Des matériaux et des problèmes abordés dans son premier ouvrage (Dios aisa) polarisent un thème sur lequel reviendront plusieurs contributions ultérieures : l'origine des dieux et des hommes, et leurs destinées respectives (théogonie et cosmogonie). Dès l'article pionnier de 1953-1954, travail préparatoire à un volume général (Teogonie e cosmogonie, Roma, 1960), qui, posant des distinctions typologiques fondamentales, compare la Théogonie d'Hésiode aux récits suméro-akkadien et hourrito-hittites, à Protogonos (1957), qui introduit la théogonie orphique par rapport aux cosmogonies phénicienne et égyptienne, et aux développements mithraico-gnostiques, à Prometbeus (1961), qui insiste surtout sur les thèmes du démiurge-trickster, du péché originel dans un cadre dualiste quoique olympien, du rôle d'elpis dans et hors du pithos, à Razza aurea (1963) (dont Aspetti aurei [1968] est un corollaire à un niveau socio-politique), qui est un contrepoint sur l'ambivalence des conditions des origines, visant autant les hommes que les dieux et se répercutant sur l'éthique et l'eschatologie, on en arrive aux retractationes de la maturité : deux conférences, en anglais (1987) et en italien (1987), et les subtiles «variazioni sul tema » de 1988. Voici le leitmotiv de cette espèce de «théologie de l'histoire » esquissée par Bianchi : le caractère ascendant (ou évolutif) des théogonies grecques (en opposition au caractère descendant des généalogies divines du gnosticisme); le théopantisme, plutôt que panthéisme, orphique; la gradation du divin dans un cadre polythéiste; la théodicée de Platon et des platoniciens, en tant qu'interrogation sur la bonté de dieu, la liberté de l'homme et l'origine du mal (Leibniz).

Les espérances des initiés aux Mystères d'Éleusis, qui représentent le paradigme des mystères, en contraste avec la mystériosophie (typiquement dualiste, anticosmique et antisomatique) des Orphiques, voilà un autre des thèmes privilégiés par Bianchi. Après une étude où est mise en valeur l'opposition entre la «saggezza olimpica » et la « mistica eleusina » donnant une analyse pénétrante de l'Hymne bomérique à Déméter (1964), deux contributions successives offrent une lecture autant psychologique que théologique de plusieurs documents de la mystique antique (Pindare, fr. 131 et Ném. VI; Isocr., Panég. 28 [1972]; Plut., De anima, fr. 178 [1974]). Le secret même des mystères, le sens final et complet de la gnose mystérique se trouve dans la tension « qui par la foi et l'espérance rapproche les termes extrêmes de la vie et de la mort, et anticipe la mort avec tout ce qu'elle portera avec elle » (Ho sympas aion, p. 286).

Le troisième - et le plus important - pôle de la recherche de Bianchi, avant et après son livie capital $I l$ dualismo religioso (Roma, 1958), est celui des doctrines dualistes par rapport à la mystériosophie et à la métaphysique platonicienne. L'orphisme tout d'abord. Si, dans le Protogonos (1957), on décèle le caractère moniste (théopantiste plutôt que panthéiste) des théogonies orphiques situées dans un contexte dualiste - en analogie parfaite avec les idées du gnosticisme -, dans le c.r. de l'ouvrage critiquable de L. Moulinier (1957) et dans un contribution ultérieure (1974), il revendique l'existence de 
l'orphisme en tant que ethos ou religiosité dualiste qui a profondément marqué la vie spirituelle des Grecs (Pythagore et Empédocle avant Platon), dans le sens de l'idée que l'homme est caractérisé jusque dans les racines profondes et divines de son être par une ambiguité congénitale, qui qualifie le type de salut auquel il aspire (le clivage ontologique qui est la cause de cette ambiguîté et qui dépend d'un "péché antécédent », préhumain, donnant lieu à une expression radicale de dualisme, au moins à un niveau anthropologique). Après Orphée, mais véritable clé de voûte de tout le système, voici Platon, avec son élève Plutarque, dualiste et en même temps interprète acharné de dualismes. Dans une série de contributions en italien (1987), en anglais (1987) et allemand (1987 et 1993), Bianchi s'attaque à des passages classiques de la République, du Timée, du Théétète, du Politique, des Lois, afin d'éclairer les liens entre théodicée et dualisme radical dans la vision de Platon. De cette investigation il ressort qu'il existe une dialectique irréductible entre l'intelligible et le sensible, entre l'unité et la multiplicité, entre l'idea et la chora, entre les deux âmes du monde et entre les deux mouvements alternatifs du cosmos. La démonologie du platonisme moyen (Plutarque) et du néo-platonisme (Plotin) enfin, envisageant à la suite de Xénocrate la présence mutuelle de démons bons et de démons méchants, intègre la théorie des démons dans la théorie du mal se référant à une ontologie dualiste ( $c f$. l'article de 1990).

Cette investigation de faits historiques aboutira bientôt à une typologie historique de la religion mystique de la Grèce, délimitée depuis 1964, développée dans les deux volumes de 1976 et dans les remarques préliminaires de deux colloques, consacrée enfin dans un exposé sommaire en anglais (1989) et dans une synthèse de vulgarisation pour le Trattato di antropologia del sacro (1992), qui va bientôt paraitre en français. Cette typologie lui tenait beaucoup à cour parce que, non seulement, elle fixait des distinctions rigoureuses à l'intérieur d'un monde chaotique, mais elle insérait aussi une expérience cultuelle grecque dans les cadres généraux de l'histoire des religions. La catégorie «mystique " (spécialement le dionysisme) impliquait le monde des rites de fertilité (et des dieux sujets à des crises ou vicissitudes); celle de « mystérique » (spécialement Éleusis) comportait la référence aux initiations de niveau ethnologique; celle de "mystério-sophique" (spécialement l'orphisme) donnait lieu à la notion générale de «péché antécédent » et à celle encore plus importante de dualisme. De plus, la catégorie la plus compréhensive, celle de religion mystique, impliquant une interférence entre niveau divin et niveau humain, se définit en opposition à celle d' "olympien ", qui refuse cette interférence. Les deux catégories s'excluent mutuellement, comme il ressort de l'analyse très fine concernant le couple des Dioscures, dieux soteres de type olympien, opposés à deux divinité soteres de type mystique comme Déméter et Coré (1987).

Enfin, quelle leçon - en ce qui concerne la religion grecque - nous a laissé en héritage Ugo Bianchi ? Une leçon importante qui s'articule à plusieurs niveaux. Primo, il nous a enseigné - comme Pettazzoni et Brelich - qu'on ne 
peut étudier la religion grecque en l'isolant des autres mondes religieux (et cela fait de lui un historien des religions et - considérant l'ensemble de son ouvre un des plus éminents de ce siècle). Secundo, il ressort de son enseignement qu'il faut bien sûr connaître les détails philologiques, archéologiques, historicolinguistiques, mais aussi prendre ses distances à leur égard. Il faut par contre tirer des conclusions en se fondant sur des données établies et chercher le sens profond d'un phénomène en le rapportant à la structure de fond (et cela fait de lui un véritable philologue de la religion, bien qu'il ait toujours eu des problèmes avec les philologues spécialisés; et un véritable phénoménologue de la religion - avec un voile de philosophie et de théologie -, bien qu'il refusât fermement cette étiquette). Tertio, il a lutté avec une ardeur inlassable en faveur d'une nomenclature rigoureuse. Avant de comparer (et ensuite mêler) des phénomènes divers, il faut les distinguer et donner des définitions claires, cohérentes et polyvalentes en même temps, des concepts qu'on utilise, définitions qui ne doivent pas résulter d'idées a priori mais du sondage historique (il définissait cette méthode par la formule «typologie historique »). Bien entendu, l'application d'une telle méthode à l'étude de faits historiques concrets se révèle complexe. D'un point de vue logique, un type historique, un universel concret est une contradictio in terminis. Une «thématique » (un mot clé pour Bianchi, et parfois une abstraction qui occulte la variété des phénomènes historiques) dans certains cas existe surtout pour celui qui l'a imaginée, mais assez mal pour les historiens spécialistes de cultures spécifiques qui connaissent bien la complexité infinie et contradictoire des créations humaines, en Grèce comme ailleurs. N'étant pas dogmatique, il s'appliquait à mettre à l'épreuve ses "thématiques», convoquant des équipes de savants pour confronter des expériences diverses. À dire vrai, il en résultait parfois une espèce de concordia discors, ou, pire, un dialogue de sourds.

Pour conclure, un souvenir personnel me semble révélateur. En 1983, revenant d'une visite à Zürich, je lui annonçai avec une certaine excitation qu'une édition pirate (mais presque complète) du papyrus de Derveni venait de sortir. Il ne parut pas très touché et, souriant, me confia : « Mais dans le papyrus de Derveni, il n'y pas d'orphisme !». Devant ma stupeur, il précisa : « Mais non, il n'y a pas la doctrine du soma-sema, qui est le signe du véritable orphisme ». En 1989, revenant d'un colloque à Genève, je lui montrai le texte des lamelles de Pelinna qui venaient d'être publiées dans Hellenika, 1987. Je vis ses yeux s'illuminer et les signes d'une émotion profonde se manifester dans toute sa personne à mesure que se poursuivait sa lecture. Il avait décelé les traces évidentes d'une doctrine de salut portant l'empreinte du dualisme et de la mystériosophie (Cf. Misteri di Eleusi, dionisismo, orfismo, p. 277 et n. 11). 


\section{Bibliographie de Ugo Bianchi se rapportant à la religion grecque}

\section{Livres}

$\Delta \mathrm{tò} \varsigma \alpha \hat{i} \sigma \alpha$. Destino, uomini e divinità nell'epos, nelle teogonie e nel culto dei Greci, Roma, 1953

La religione greca, Torino, 1975

The Greek Mysteries, Leiden, 1976

Prometeo, Orfeo, Adamo. Tematiche religiose sul destino, il male, la salvezza, Roma, 1976

\section{Articles}

Teogonie greche e teogonie orientali, in SMSR, 24-25 (1953-1954), p. 60-75

Protogonos. Aspetti dell'idea di Dio nelle religioni esoteriche dell'antichità, in SMSR, 28 (1957), p. 115-133

La dea di Lindos, in Epigraphica, 19 (1957), p. 10-24

Orfeo e l'orfismo, in SMSR, 28 (1957), p. 151-156

Prometheus, der titanische Trickster; in Paideuma, 7, 8 (1961), p. 414-437

Razza aurea, mito delle cinque razze e Elisio, in SMSR, 34 (1963), p. 143-210

Saggezza olimpica e mistica eleusina nell'inno a Demetra, in SMSR, 35 (1964), p. 161-193

Aspetti aurei della regalità greca, in Atti e memorie del I Congresso internazionale di Micenologia, Roma, 1968, p. 1040-1045

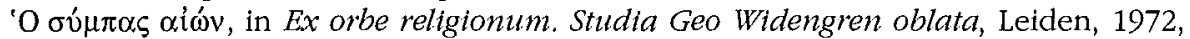
I, p. $277-286$

L'orphisme a existé, in Mélanges d'Histoire des religions offerts à Henri-Charles Puech, Paris, 1974, p. 129-137

Eleusis, Stob., IV, p. 1089 H. et Plat., Pbaedr. 248 A-B, in Mélanges de Pbilosopbie, de Littérature et d'Histoire anciennes offerts à Pierre Boyancé, Roma, 1974, p. 73-77

Per la storia della teologia dei Greci: la Teogonia di Esiodo, in La coscienza religiosa del letterato pagano, Genova, 1987, p. 9-26

Plutarch und der Dualismus, in ANRW II, 36.1 (1987), p. 350-365

The Ideal Foundations of Greek Cosmogonical Thought, in Mythology and Cosmic Order; Helsinki, 1987, p. 9-15

I Dioscuri: una versione della coppia divina, in SMRS, 53 (1987), p. 41-56

Il 'prima' e l'altrove'. Variazioni sul tema del rapporto tra dei e uomini nella religione greca antica, in Kernos, 1 (1988), p. 9-17

Mystery Cult and Gnostic Religiosity in Antiquity, in J. PODEMANN SøRENSEN (éd.), Rethinking Religion. Studies in the Hellenistic Process, Copenhagen, 1989, p. 11-22

Die "Mittlere Stoa" und ibr Publikum, in P. ANTES, D. PAHNKE (éds), Die Religion von Oberscbicbten, Marburg, 1989, p. 13-22

Sulla demonologia del medio- e neoplatonismo, in E. Corsini, E. CosTA (éds), L'autunno del diavolo, Milano, 1990, I, p. 51-62

Misteri di Eleusi, dionisismo, orfismo, in J. RIES (éd.), Trattato di antropologia del Sacro, 3 : Le civiltà del Mediterraneo e il Sacro, Milano, 1992, p. 259-281

Ein Beitrag zur Geschichte des Dualismus in Griechenland, in $Z R W, 1$ (1993), p. 3-12

\section{Comptes rendus}

de W. Burkert, Griechiscbe Religion..., in Gnomon, 53 (1981), p. 417-425

de W. Burkert, Antiken Mysterien, in Gnomon, 67 (1995), p. 1-5. 